\title{
Figur: Zu einem Kernproblem historischer Narratologie
}

\section{I. ,Historische Narratologie}

In ihrem programmatischen Beitrag The Diachronization of Narratology von 2003 hat MONIKA FLUDERNIK gezeigt, welche Randstellung die Geschichtlichkeit des Erzählens als Forschungsobjekt in der klassischen Narratologie einnahm. ${ }^{1}$ Zurückzuführen ist dies - selbstverständlich, möchte man fast sagen - auf die synchronische Ausrichtung der Narratologie, sei sie strukturalistischer Prägung oder Modellen möglicher Welten verpflichtet. ${ }^{2}$ Für FLUDERNIK ist eine Diachronisierung als ,exciting new area of research" (S. 332) das Gebot der Stunde, eine neue Richtung also, der ihr zufolge drei Forschungsinteressen den Weg bereitet haben: das feministische rewriting von kanonischer Literaturgeschichte mit dem Ziel, Literatur und Erzählmodelle von und für Frauen wiederzuentdecken, die Ausweitung des narratologischen Interesses auf Geschichtswerke (in der Nachfolge HAYDEN WHITEs) und die Forschungen zu Vorformen und Entstehung des neuzeitlichen Prosaromans (S. 331f.). ${ }^{3}$

1 MONIKA Fludernnk: The Diachronization of Narratology. In: Natrative 11 (2003), S. 331-348.

2 Hict' S. 331: "Classical structuralist narratology, with its, geometrical imaginary" (Gibson), necessatily had a synchronic outlook, and the same is true for possible world theory and many poststructuralist approaches." FLUDERNIK bezieht sich auf ANDREW GIBSON: Towatds a Postmodern Theory of Natrative, Edinburgh 1996.

3 Vor allem die feministisch akzentaierte Nartatologic hat den Gegenstandsbereich der klassischen Narratologie nicht nur ausgeweitet, sondern diese auch politisiert. Zur Diskussion um die Zuständigkeiten und Grenzen der Narratologie angesichts dieser neueren Kontextualisietungen siehe bes. die Diskussion zwischen DAVID DARBY und TOM KINDT/HANS-HARALD MOLLER: DAVID DARBY: Form and Context. An Essay in the Flistory of Narratology. In: Poetics Today 22 (2001), S. 829-852; TOM KINDT/HansHARALD MULLER: Narratology and Interpretation. A Rejoinder to David Darby. In: Poetics Tuday 24 (2003), S. 413-421; DAVID DARBY; Form and Context Revisited. In: Poetics Today 24 (2003), S. 423-437; siehe auch TOM KINDT/HANS-HARALD MÜLLER: Narrative Theory and/or/as 'Theory of Interpretation. In: What is Narratology. Questions and An- 
Im skizzierten Programm einer diachronischen Narratologie identifiziert FLUDERNIK mehrere Bereiche, die sich für diachronisch ausgerichtete erzähltheoretische Fragen eignen würden: etwa historische Differenzen in der Ausgestaltung der Etzählfunktion, die histotische Bedeutung und Funktion metanarrativer oder metafiktionaler Passagen, die Behandlung und Ausgestaltung orientierender Anfangspassagen in Erzählungen, oder auch Fragen zur geschichtlichen Spezifität aller Elemente klassischer Narratologie: Fokalisierung, Person (etwa das historische Auftreten und die Entwicklung der Ich-Erzählung), Zeitbehandlung, Einführung und Beschreibung von Orten und Szenerien oder von Figuren. Die Liste, so FLUDERNIK, sei fast unendlich, denn so wenig sei aus narratologischer Sicht über historische Aspekte des Erzählens gearbeitet worden (S. 334).

FLUDERNIKs Beitrag kommt in einer Zeit, die ein bemerkenswerter Wandel in der allgemeinen Narratologie wie der Altgermanistik prägt. In

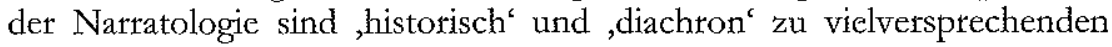
Adjektiven zur Verortung jeweiliger Modelle geworden, neben FLUDERNIK sei hier auf Arbeiten von FOTIS JANNIDIS und ANSGAR NÜNNING verwiesen. ${ }^{4} \mathrm{Zu}$ beobachten ist also, dass Studien mit systematischem Charakter diachronische Aspekte aufnehmen. Gleichzeitig werden in der diachronen Literaturwissenschaft, als die sich die Altgermanistik unter anderem versteht, schon seit einiger Zeit narratologische Modelle zur Textbeschreibung und Analyse angewendet. Besonders wichtig sind in diesem Zusammenhang JAN-DIRK MÜLLERs Spielregeln für den Untergang, das unter anderem die Konstitution des Helden in der Heldenepik zum Thema hat, ${ }^{5}$ und GERT HÜBNERs Erzäblform im böfischen Roman, in dem er die Modelle und Beschreibungssprache der Narratologie GÉR $\Lambda$ RD GENETTEs auf hochhöfische Epen anwendet. ${ }^{6}$ Mein Versuch schließt hier an. Ich möchte Fragen der Historisierung der Narratologie in einem Feld durchspielen, das historisch besonders voraussetzungsreich ist und daher spezielle Herausforderungen bietet: die Konturierung der Figur im höfischen Roman.

swets Regarding the Status of a Theory. Hrsg. von DENS., Berlin, New York 2003 (Narratologia 1), S. 205-219.

4 FOTIS JANNIDIS: Figur und Person. Beitrag zu einer historischen Natratologie, Berlin, New York 2004 (Natratologia 3); ANSGAR NÜNNING: Towards a Cultural and Historical Narratology. A Survey of Diachronic Approaches, Concepts, and Research Projects. In: Anglistentag 1999 Mainz. Proceedings. Hrsg. von BERNHARD REITZZ/SIGRID RIEUWERTS, Trier 2000, S. 345-373.

5 JAN-DIRK MÜLLER: Spielregeln für den Untergang. Die Welt des Nibelungentiedes, Tübingen 1998.

6 GERT HÜBNRR: Erzählform im höfischen Roman, Studien zur Fokalisierung im Eneas, im Iwein und im Tristan, Tübingen 2003 (Bibliotheca Getmanica 44). 


\section{Zu einer historischen Nartatologie der Figur}

Die literatische Figur als solche spielt in allgemeinen narratologischen Modellen im Vergleich etwas zur Behandlung von Zeit und Perspektive, nicht die Hauptrolle: ${ }^{7}$ in der inzwischen für den deutschsprachigen Raum kanonischen Einführung in die Erzähltheorie von MICHAEL. SCHEFFEL und MATÍAS MARTÍNEZ etwa gibt es keine eigene Behandlung der Figur, und im Appendix dieser Einführung keinen Eintrag zur Figur. ${ }^{8}$ Solche Niedriggewichtung hat vielerlei Ursachen, aber die primäre ist wohl in der Geschichte der Narratologie selbst zu finden. Dem Formalismus galt die Figur als sekundär und wurde vor allem als Orientierungsgröße innerhalb der Handlung, als Hilfe zur Klassifizierung und Orientierung wahrgenommen bzw. in der folgenreichen Theorie VIADIMIR PROPPs lediglich als Verweis auf eine tiefer liegende invariante Aktantenstruktur gelesen. ${ }^{9}$ In diesem Sinne, nämlich als Aktualisierung eines tiefenstrukturellen Aktanten, wurde die konkrete Ausgestaltung der Figur als Oberflächenphänomen klassifiziert. Diese funktionalistische Sicht wurde im Strukturalismus von ALgIRDAS JULIEN GREIMAS fortgeschrieben. ${ }^{10}$ Selbst bei GENETTE, der mit der Frage, wer sieht' den Figuren implizit einen wichtigen Platz einräumt, geht es eben kaum um die Figur, sondern ihren Blick, ihre Perspektive, ihre Rolle in der Geschehensvermittlung. ${ }^{11}$

7 So auch aus mediävistischer Perspektive CIIRISTOPHER YOUNG: The Chatacter of the Individual in Hartmann von Aue's Erec. In: Arthurian Literature 16 (1998), S. 1-25, hier S. 11. Für wichtige systematische Vorstöße siehe URI MARGOI.IN: Characters and Their Versions. In: Fiction Updated. Theories of Fictionality, Narratology, and Poetics. Hrsg. von CALIN-ANDREI MiHALIESCU/WhLID HAMARNEil, Toronto, Buffalo 1996, S. 113-132; DERS.: ,Character. In: Routledge Encyclopedia of Natrative Theory. Hrsg. von DAVID HERMAN/MANFRED JAHN/MARIE-LAURE RYAN, $\Lambda$ bingdon, New York 2005, S. 52-57; NLEX WOLOCH: The One vs. the Many. Minor Characters and the Space of the Protagonist in the Novel, Princeton, Oxford 2003 (Diskussion zur narratologischen Behandlung der literatischen Figur cbd. S. 14-21, mit weiterer Literatur). Grundlegend für die narratologische Perspektive auf literarische Figuren sind SEYMOUR CHATMAN: Story and Discourse. Narrative Structure in Fiction and Film, Ithaca, London 1978; JAMES PHEJ.AN: Reading People, Reading Plots. Chatacter, Progression, and the Interpretation of Narrative, Chicago 1989; DAvid Herman: Story Logic. Ptoblems and Possibilities of Nartative, Lincoln, London 2002, S. 115-169. Weitere Hinweise bei GÖRAN NIERAGDEN: ,Figurendarstellung, literarische. In: Metzler Lexikon Literatur- und Kulturtheorie. Ansätze - Personen - Grundbegtiffe. Hrsg. von ANSGAR NÜNNING, Stuttgart, Weimar 22001, S. $175 f$.

8 MATías MARTÍNEZ/MICHAEt, SCHEFFEL: Einführung in die Etzähltheorie, München 1999.

9 VLADIMIR PROPP: Morphologie des Märchens. Hrsg. von KARL ETMERMACHIER, München 1972.

10 ALGIRIDAS JULIEN GREIMAS: Strukturale Semantik. Methodologische Untersuchungen, Braunschweig 1971 (Wissenschaftstheorie, Wissenschaft und Philosophic 4).

11 GÉrARD GENETrTE: Die Erzählung. Aus dem Trz. von Andreas Knop, mit einem Nachw. hrsg. von JOCHEN VOGT, München 21998. 
Früh aber meldeten sich Zweifel an der strukturalistischen Sichtweise. So mahnte SHLOMITH RIMMON-KENAN die „Ausarbeitung einer systematischen, nicht reduktiven aber auch nicht impressionistischen Theorie der Figur"12 an. SEYMOUR CHATMAN lehnte in Story and Discourse von 1978 den reduktiven Funktionalismus der Strukturalisten ab. Seine „offene Theorie der Figur."13 ist stark auf moderne literarische Texte hin orientiert und lässt sich vor allem aufgrund der anderen Mimesiskonventionen nur schwer auf mittelalterliche Texte anwenden: CHATMAN geht es um im weiteren Sinne realistische Figurendarstellungen. Seine Diskussion bietet aber wichtige Impulse, vor allem den, dass Figuren - weit davon entfernt, bloße Handlungserzeuger oder Anker zur Orientierung im Handlungsgeschehen zu sein - als Größe der Etzählwelt eine zentrale Stellung beanspruchen können und dass sie ein gewisses Eigengewicht gegenüber der Handlung einnehmen, etwa unabhängig von verbaler Gestaltung und Handlungabfolge erinnerbar sein: "Too often do we recall fictional characters vividly, yet not a single word of the text in which they came alive" (S. 118). Zu dieser Protagonistenbezogenheit der Literaturerinnerung finden sich auch für die mittelhochdeutsche Literatur genügend belegende Beispiele. So ließe sich die in der mittelhochdeutschen Literatur weithin sichtbare Gewohnheit anführen, Texte, auf die referiert wird, mit dem Namen des oder der Protagonisten zu bezeichnen. Auch metanarrative Passagen legen nahe, dass Protagonisten einen herausgehobenen Status haben, z.B. wenn Parzival bei Wolfram als des mares hêrre bezeichnet wird. ${ }^{14}$ In lehnsrechtliche Terminologie gebracht ist damit nicht nur die größere Bedeutung Parzivals gegenüber Gawan, sondern auch die Sekundarität von Handlungsverlauf und -gestaltung gegenüber der sie bestimmenden Figur.

Die Probleme der narratologischen Theoriebildung in Bezug auf die Figur begininen beteits bei der Platzierung der Größe literarische Figur innerhalb der Basisdifferenz discours und histoire, die zuerst eine Differenz der Zeitbehandlung, Kausalisietung und Perspektivengestaltung ist und als solche in verschiedenen Erzähltheorien jeweils leicht unterschiedlich und auch, etwa in TOMAŠEVSKJJs Unterscheidung von Fabel und Sujet, früh

12 SHLOMITH RIMMON-KENAN: Natrative Fiction. Contemporary Poetics, London 1983: "the elaboration of a systematic, non-reductive but also non-impressionistic theory of character remains one of the challenges poetics has not met yet." In der überarbeiteten zweiten Auflage ihres Standardwerks (London 2002) kann RIMMON-KKNAN die Forderung unvetändert wiederholen.

13 CHATMAN (Anm. 7), S. 119: „Open theory of character“.

14 Zitiert nach: Wolfram von Eschenbach: Parzival. Nach der Ausgabe KArL LACHMANNs revidiert und kommentiert von EBERHARD NELLMANN, übertragen von DIETER KÜHN, Frankfurt a. M. 1994 (Bibliothels deutscher Klassiket 110; Bibliothek des Mittelaltets 8), hier 338,7 . 
theoretisiert wurde: die Fabel ist „die Gesamtheit der Motive [einer Erzählung] in ihrer logischen, kausal-temporalen Verknüpfung" und das Sujet "die Gesamtheit derselben Motive in derjenigen Reihenfolge und Verknüpfung, in der sie im Werk vorliegen." ${ }^{15}$ Das funktioniert sehr gut, weil Zeit und Kausalität nicht nur Basiskomponenten des Erzählens, sondern auch ausreichend abstrahierbare Konzepte sind. Es funktioniert auch für Perspektive und Fokalisierung, da man sie als Abweichungen von einer Nullstufe wahrnehmen kann. In der Erforschung von beidem, narrativer Zeitgestaltung und Kausalisierung sowie Folsalisierung verbuchte die Narratologie ja auch ihre wichtigsten Erfolge; hier produzierte sie quasi ihre Exportschlager über den eng gestrickten, begrifflich manchmal exklusiven und institutionell relativ geschlossenen Eigenbereich hinaus. Raum bzw. setting und Figur, die zwei weiteren entscheidenden Größen für die Konstitution einer Erzählung, dagegen sperren sich der Zuordnung. JANNIDIS stellt daher zurecht fest, dass für Figuren Formen von Sequenzierung, sozusagen die idealisierte Nullstufe, wie man sie für Zeit und Kausalität in der bistoire (re)konstruieren kann, nicht einfach ermittelbar sind. ${ }^{16}$ Sicherlich ist es so, dass man erzählte Figuren auf beiden Ebenen ansetzen muss: eben nicht nur als Träger der Handlung auf der bistoire-Ebene, sondern als spezifisch Gestaltete auf der discours-Ebene: als signifikant beschriebene und erzählerisch spezifisch ausgestattete Figuren, bei deren Darstellung eine reduktive und allusive Technik zum Tragen kommen muss. Damit will ich aber nicht sagen, dass die Figur nicht, genau wie das setting, ein Basiselement der bistoire sein muss, vor allem dann, wenn man bistoire grundsätzlich von ihrem zeitlich-kausalen Charakter her denkt: Figuren und Ort sind Grundkonstituenten des Erzählens, die allererst Handlung ermöglichen.

Im Modell von GREIMAS wäre wegen des spezifischen Zuschnitts seiner Erzählgrammatik keine Schwierigkeit bei einer Ebenenzuordnung aufgetreten. Die Figuren hätte man dem discours oder der parole zuordnen können, da sie in diesem Modell lediglich die Aktanten der Tiefenebene, der narrativen langue, vertreten. ${ }^{17}$ Mit der Einsicht, dass die Aktantenstruktur zu stark reduktiven Ergebnissen führt und das Ptojekt einer universalen Erzählgrammatik als gescheitert gelten kann, verlor man aber mit der

15 BORIS TOMAŠEVSKKI: Theorie der Litesatur - Poetik. Nach dem Text der 6. Aufl. (Moskau - Leningrad 1931). Hrsg. von KLAUS-DIEITER SEJEMANN, Wiesbaden 1985 (Slavische Studienbücher N.F. 1), S. 218; siehe auch VIKTOR ŠKLOVSKIJ: Det parodistische Roman. Sternes Tristram Shandy. In: Texte der tussischen Formalisten. Hrsg. von JURIJ STRIEDTER, Bd. 1, München 1969 (Theorie und Geschichte der Literatur und der Schönen Künste 6,1), S. 245-299, hier S. 297 und 299. Zu konkursierenden Modellen vgl. MAR'IT́NEZ/SCHEITEL ( $\Lambda$ nm. 8), S. 20-26 und 108-111.

16 JANNIDIS ( $\Lambda$ nm. 4), S. 164-166.

17 Diskussion bei HeRMAN $(\Lambda \mathrm{nm} .7)$, S. 115-169. 
langue die für die Figur postulierte Nullstufe, den Aktanten, und damit ebenjenen Nullpunkt der Orientierung, der für Zeit und Kausalität in narratologischen Modellen aufrechterhalten werden konnte. ${ }^{18}$ Die Schwierigkeiten mit dem narratologischen Ort der Figur scheinen zum Teil damit zusammenzuhängen, dass dieses Projekt einer Erzählgrammatik weitgehend aufgegeben wurde, die Narratologie sich aber ihrer strukturalistischen Wurzeln nicht entledigen kann.

Wie steht es mit der Historisierung des Konzepts Figur? JANNIDIS identifiziert in seiner sehr grundsätzlichen narratologischen Behandlung der Figur gewisse Basisannahmen, die für die Figur in Anschlag zu bringen sind. Er fasst das in seiner tezeptions- und kognitionsorientierten Theorie so,

dass Leser zumeist ohne Probleme erkennen, dass eine Figur eine Figur ist und kein Objekt. Daran läßt sich zeigen, dass die Kategorie ,Figur' prototypisch organisiett ist, d.h. wie prototypisch eine Figur ist, läßt sich durch den Bezug auf die Merkmalskombination in einem besten Exemplar bestimmen. Dieses beste Exemplar für die Kategorie der Figur ist wohl eine im Äußeren menschenförmige und im Inneren den jeweiligen kulturellen Konzepten von typisch menschlichen mentalen Zuständen konforme Gestalt. ${ }^{19}$

Dies ist nun sehr grundsätzlich und eher systematisch als historisch angelegt: Bei jedem Versuch der Historisierung dieses Konzepts wird man schnell darauf stoßen, dass die dafür entscheidende Operation die Identifizierung jener offen gelassenen ,jeweiligen kulturellen Konzepte“ ist und zwar im übrigen, was für das vormoderne Erzählen entscheidend zu sein scheint, nicht nur Konzepte des Körperinneren, sondern auch des Körperäußeren. Diese Identifizierung wird bei JANNIDIS dem generischen und dem Weltwissen des Lesers zugeordnet: Historisch konkretisierte Umrisse dieses Wissens, selbst wenn es nur innerhalb eines Textes oder einer Textgruppe ermittelt wird, wären notwendig, um überhaupt von einer historischen Narratologie zu sprechen. Hier liegt ein gewisses Problem in JANNIDIS Entwurf: wo man, dem Untertitel des Buches nach, historische Konkretisation erwarten würde, verbleibt das Modell weitgehend im Ahistorisch-Systematischen. In einer historischen Narratologie müssten also die ,jeweiligen kulturellen Konzepte“ - textuelle und kulturelle Anthropologien, generisches Wissen über Figuren, intertextuelle Sinnkonstitutionen - stärker in die nattatologische Modellienung eingebracht werden, als JANNIDIS dies tut. Dennoch wird man schon zugeste-

18 Ganz unbestritten ist aber auch für die 7eit die Gültigkeit der Unterscheiơung zwischen bistoire und discours nicht; bereits B $\Lambda$ CHTINs Chronotoposmodell kann als ein Gegenentwurf dazu angesehen werden. S. dazu jetzt JON $\Lambda$ THAN STONE: Polyphony and the Atomic Age. Bakhtin's $\Lambda$ ssimilation of an Einsteinian Universe. In: PMLAA 123 (2008), S. 405-421, hier S. $412 \mathrm{f}$.

19

JANNIDIS (Anm. 4), S. 239. 
hen, dass Figuren auf ein dem jeweiligen historischen Verständnisrahmen angepasstes anthropomorphes bestes Exemplar hin entworfen sind.

Ein zweites Problem, das sich mit der Figur in ihrer Position in einem narratologischen Modell verbindet, betrifft das Verhältnis von Figur, setting und Handlungsabfolgen, das FuUDERNIK problematisiert hat. Sie geht dabei von CHATMANs Unterscheidung von events und existents aus. Existents bezeichnen bei CHATMAN die da-seienden, statischen Elemente einer Geschichte, events die handlungs- und damit letztlich zeiterzeugenden dynamisierenden Elemente der Geschichte. ${ }^{20}$ FLUDERNIK befragt diese Unterscheidung und stellt Ansätze einer Narratologie vor, welche die Figur zum methodischen Angelpunkt macht:

Die Ebene der Geschichte wird in den meisten Erzähltheorien einmal axiomatisch als Kombination von Schauplatz (setting) und Charakteren (actants) dargestellt. Paradoxerweise sind so die Basiselemente traditioneller Geschichte nur existenziell, daher statisch veranlagt. Charaktere und Setting figurieren im Exzähltext meist als Beschreibungspassagen. Erst durch Handlungsabfolgen - durch die Handlungen der Figuren - entsteht die zeitliche Grundkonstellation. So unterscheidet Chatman auf der story-Ebene zwischen events und existents. Sieht man Charaktere allerdings als prototypisch menschlich an, dann kann man ihre Handlungen als Teil ihrer existenziellen Befindlichkeit erfassen und auch den Schauplatz dynamisch sehen, nämlich als Umwelt, die Ereignisse und Entwicklungen, die von außen auf die Protagonisten einwirken, inkludiert. ${ }^{21}$

Dies ist eine fundamentale Rückbesinnung auf das anthropomorphe Element der Figur, wie sie auch bei JANNIDIS anklingt: in dieser Perspektive wird das Gewicht des Handlungsverlaufs von der Handlungsstruktur wegverlagert und als Teil einer fundamentalen Charakteristik von Figuren verstanden. Anders gesagt: eine Figur, eine Grundbedingung des Erzählens, lässt immer Handlung erwarten. Da dies zum prototypisch Menschlichen der Figur gehört, wird dieser Zusammenhang gxundsätzlich erzähltheoretisch relevant sein. Diese Lösung würde nun also die Figur, nicht Handlungs- bzw. Erzählschema ins Zentrum stellen. Diese anthropozentrische Lösung, wie ich sie etwas ungeschützt nennen will, entspricht einer gewissen Intuition historischer Gegebenheiten und kommt eben auch der historisch nachweisbaren zentralen Position der (Haupt-)Figur in der Textwahrnehmung durch Autoren und Rezipienten entgegen. Weithin ungelöst bliebe jedoch weiterhin die Frage, welche anthropologischen Interferenzen an der Figurenkonzeption teilhaben.

Hier liegt die entscheidende Herausforderung an eine historische Narratologie der Figur, ganz abgesehen davon, dass hier auch ein großes Problem der allgemeinen narratologischen Theoriebildung zur Figur liegt.

20 Vgl. Chataman (Anm. 7), S. 44-145.

21 MONIKA FLUDERNIK: Einführung in die Erzähltheorie, Darmstadt 2006, S. 41. 
Das hängt vor allem mit der Komplexität zusammen, die sowohl die literarische Gestaltung als auch die konzeptionelle Verarbeitung von Figuren durch Rezipienten prägt. In beiden Fällen nämlich wird die textuelle Konstruktion Figur stärker, als dies bei anderen Elementen der Erzählwelt der Fall ist, mit anthropomorphen Interferenzen konfrontiert. Für einen historischen Zugriff auf das mittelalterliche Erzählen, dies wird etwa in der Diskussion der Figur in Heldenepik und Roman der mittelhochdeutschen Literatur deutlich, die JAN-DIRK MÜLLER in seinen beiden großen Monographien vorgelegt hat, besteht die entscheidende Frage darin, wieweit etwa handlungspsychologische Annahmen in Anschlag gebracht werden können, um Figur, ihre Konzeption und Motivation zu erklären. Historisierung meint hier die methodisch kontrollierten Beschreibung einer textuell-generischen Anthropologie, die nicht unbedingt durch riskante $\mathrm{Be}$ züge auf fremdtextliche Diskurse abgesichert werden muss, sondern die aus dem Text ${ }^{22}$ oder Textgruppen ${ }^{23}$ selbst heraus entwickelt werden soll. Dabei wird versucht, die Gefahr überzeitlich-ahistorischer Konstrukte durch methodische Reflexion zu bannen: Mit MÜLLER

ist mit der Möglichkeit historisch andersartiger Entwürfe von psychischen Dispositionen, Verhaltensmustern, Motivationen und personaler Identität zu rechnen. Diese zu ermitteln, stößt freilich auf erhebliche methodische Schwierigkeiten, vor allem, was die Quellen betrifft. Hier empfiehlt sich ein streng textbezogenes Vorgehen: Literarischen Texten sind, wie Texten auch sonst, mehr oder minder explizit anthropologische Modelle eingeschrieben. Diese Modelle sind nicht unabhängig von anderen, in andere Texte eingeschriebenen oder praktisch gelebten Modellen der gleichen Kultur, doch empfiehlt es sich, die Frage nach der Beziehung unterschiedlicher Diskurstypen und der ihnen inhärenten Modelle zueinander zunächst einmal einzuklammern. ${ }^{24}$

Wie aber könnte ein, ,nicht reduktives und gleichzeitig nicht impressionistisches" (RIMMON-KENAN) historisches Modell einer Narratologie aussehen, in der die Figur als, sprachlich erzeugte konzeptionelle Einheit" ${ }^{\text {"25 }} \mathrm{im}$ Zentrum steht? Dieses Módell müsste in die Lage versetzen, die semiotischen Verfahren des Figurenentwurfs, die Ausbildung von ,Rekurrenzmustern ${ }^{6}$ um eitien Eigennamen oder ein Appelativum herum ${ }^{26}$ sowie gleichzeitig die historisch spezifischen Spannungen zwischen Muster und anthropologischen Interferenzen zu reflektieren. Dies wäre jeweils gattungsmäßig zu spezifizieren. So bleibt etwa bei aller neuartiger „psycholo-

22 Für das Nibelhngenlied MüLLER (Anm. 5), S. 201-248.

23 JAN-DIRK MULLER: Höfische Kompromissc. Acht Kapitel zur höfischen Epik; 'Tübingen 2007, bes. S. 41-45.

24 MULLER (Anm. 5), S. 202.

25 JANNIDIS (Anm. 4), S. 147.

26 JoEI. WEINSHEMER: Theory of Character. Fmma. In: Poetics 'Today 1 (1979), S. 185-211, hier S. 195. 
gischer Charakterisierungskunst" 27 (wenn man es denn so nennen will) die schematische Modelliertheit der Figuren ein prägendes Charakteristikum des mittelalterlichen Romans. ${ }^{28}$ Figurenentwurf und auf ihn zugerichtete Handlung folgen Schemata, die generisch und wohl auch sozial vorgegeben sind (ohne dass sich diese letzte Komponente historisch klar sichern ließe). Daher würde ich bei aller Problematik dafür plädieren, belegbare Aktantenstrukturen in die Analyse einzubeziehen, ohne diese aber im Sinne einer Erzählgrammatik zu konzeptionalisieren. ${ }^{29}$ Die eigene Komplexität des Figurenentwurfs im Artusroman etwa entsteht aus einer Spannung: Figuren können wenigstens streckenweise Realisierung eines, oder manchmal auch mehrerer, Aktanten sein. Der Entwurf ist aber meist in immer neuen spezifischen Ausprägungen durch partikulare Eigenschaften bereichert. ${ }^{30}$

Eine wichtige Frage für die Bestimmung einer „textuellen Anthropo$\operatorname{logie}^{\text {" } 31} \mathrm{im}$ höfjschen Roman ist, wie sich Schematismus und Abweichung sowie unter Umständen auch unterschiedliche, konfligierende anthropologische Modellierungen zueinander verhalten, wobei diese konfligierenden Entwürfe auf unterschiedliche Figuren verteilt oder in einer Figur simultan oder subsequent realisiert sein können. Dies ist etwa dann der Fall, wenn einem schematisch bestimmten Aventiureritter über eine gewisse Textstrecke Psychonatration verweigert wird, obwohl der Text selbst diese Möglichkeit vorsieht und einer anderen Figur in derselben Textstrecke zuweist. Das geschieht in Hartmanns Erec und Enite. Hier liegt die Chance, konfligierende textuelle Anthropologien narratologisch beschreibbar zu machen. Vorbereitungen dazu sind im Rahmen von Studien zur Figuren-

27 Hartmann von Auc: Erec. Text und Kommentar. Hrsg. von MANFRED GÜNrER SCHOLZ, Frankfurt a. M. 2007 (Deutscher Klassiker Verlag im Taschenbuch 20), S. 589.

28 Ebd, vertritt SCHOLZ eine entschiedene Gegenposition.

29 So aber RALF SIMON: Einführung in die strukturalistische Poetik des mittelalterlichen Romans. Analysen zu deutschen Romancn der matière de Bretagnie, Würzburg 1990 (Epistemata 66).

30 Dabei ist zu beobachten, dass Nebenfiguren, soweit sie über die Funktion der lateralen Helferfigur hinausgehen, weniger aktanticll bestimmt zu sein scheinen: besonders deutlich wird dies im Parzival, der so reich an solchen Nebenfiguren ist. Dazu ELKE BRÖGGEN: Schattenspicle. Beobachtungen zur Erzähllkunst in Wolframs Parzival. ln: Wolftam-Studien 18 (2004), S. 171-188; MARKUS STOCK: Jähelin. Zu Figurenentwurf und Sinnkonstitution in Wolframs Parivival. In: PBB 129 (2007), S. 18-37.

31 Zum Begtiff MiCriAEI. TITZMANN: Psychoanalytisches Wissen und literatische Darstellungsformen des Unterbewußten in der Fizüben Moderne. In: Psychonnalyse in der modernen Literatur. Kooperation und Konkurrenz. Hrsg, von THOMAS ANZ, Würzburg 1999, S. 183-217; MÜI.J. (Anm. 23), S. 42, erforscht die „Bausteine zu einer Anthropologie des literarischen Helden in der Epik um 1200“. 
poetik und zur Thematisierung des menschlichen Inneren im höfischen Roman geleistet worden. ${ }^{32}$

\section{Erec und Enite}

Die mittlere Partie von Hartmanns Erec und Enite mit Krise und Auszug des Protagonistenpaares und den ersten Kämpfen des Helden stellt einen wichtigen Konzentrationspunkt der Forschung dar. ${ }^{34}$ Schwierigkeiten hat vor allem das spannungsreiche Verhältnis zwischen Erec und Enite bereitet. Narrativ werden diese Spannungen primär über die deutlich unterschiedlichen Figurenkonzepte im Entwurf von Erec und Enite erzeugt. Ausgehen will ich von der Erzählstruktur, um dann, unter Rückgriff auf einen Artikel von MARTIN JONES und auf kurze Bemerkungen zu dieser Passage in HÜBNERs Erzäblform auf die Gestaltung von Erec und Enite einzugehen. ${ }^{35}$

Die Bedeutung der sinntragenden Struktur vor allem für das Etzählen im frühen Artusroman wurde in der Folge des Modells von HUGO KUHN immer wieder betont. Die oft beschriebene, ${ }^{36}$ historisch neuartige narrative Struktur der Artusromane Chrétiens und Hartmanns (vor allem von Erec und Enite) und ihre eigentümliche, komplexe Form lassen es begründet erscheinen, mit ihrem Auftreten einen Einschnitt in der Geschichte

32 YOUNG (Anm. 7); MARITN BAISCH: Orgeluse - Aspekte ihrer Konzeption in Wolframs von Eschenbach Parzival. In: Schwierige Frauen - schwierige Männet in der Literatur des Mittelaltets. Hrsg. von Alois M. HAAS/INGRID KASTEN, Frankfurt a. M. u. a. 1999, S. 15-33; MATTHIUS MEYER: Struktut und Person im Attustoman. In: Erzählstrukturen in der Artusliteratur. Forschungsgeschichte und neue Ansätze. Hirsg. von FrIFDRICHI WOLFZETTTSL, Tübingen 1999, S. 145-163; DERS.: Der Weg des Individuums. Der epische Held und (s)ein Ich. In: Text und Kultur. Mittelalterliche Literatu: 1150-1450. Hrsg. von URSUL $A$ PETERS, Stuttigart) Weimar 2001 (Germanistische Symposien. Berichtsbände 23), S. 529-545; Inszenierungen von Subjektivität in der Literatur des Mittelalters. Hrsg. von MARTIN BAISCH u. a.; Königstein 2005; HÜBNIER (Anm. 6); MÜLL.ER (Anm. 23), S. 317-361; SILVIA REUVEKAMP: des git gawisse lêre / kïnec Artûs der guote. Zur Thematisietung und Funktionalisietung des Normativen in der Figurenpoetik des höfischen Romans. In: Norm und Normativität in der deutschen Yiteratur des Mittelalters. Hisg. von ELKE BRÜGGEN/FrANZ JOSEF. HOLZNAGEL, Berlin, New York [in Vorbereitung].

33 Zitiert wird nach der Ausgabe von SCHOLZ (Anm. 27).

34 Zusammenfassend JOACHIM BUMKKE: Der Erec Hartmanns von Aue. Eine Einführung, Berlin, New York 2006 (de Gruyter Studienbuch), S. 104-127.

35 MARTIN H. JONES: Durch schanen list er sprach. Empathy, Pretence, and Narrative Point of View in Hartmann von Aue's Erec. In: Blütezeit. Festschrift für L. Peter Johnson zum 70. Geburtstag. Hrsg. von MARK CHINCA/JOACHIM HEINZLE/CHRISIOPHER YOUNG, Tübingen 2000, S. 291-307; HÜBNER (Anm. 6), S. 402-404.

36 'Zusammenfassend KuRT RUH: Höfische Epik des deutschen Mittelalters. Bd. 1: Von den Anfängen bis zu Hartmann von Aue, 2., verb. Aufl., Berlin 1977 (Grundlagen der Germanistik 7), S. 115-141. 
der literarischen Sinnstiftung zu verbinden. ${ }^{37} \mathrm{Im}$ Artusmodell ist die zweiteilige Handlungsführung mit einem bis in Einzelheiten reichenden Netz. von Korrespondenzen und Oppositionen verknüpft. Diese Struktur hat sinnstiftende Potenz, die sich aus dem Miteinander von Analogie und Addition der Textelemente ergibt. ${ }^{38}$

In diesem Strukturdenken erklärt sich Handlung aus dem Schema, die Thematisierung einer Handlungsmotivation ist nicht unbedingt notwendig. Die Theorie der Symbolstruktur postuliert einen eigenen Typus von Protagonisten: er erfullt das Schema, ob er reflektiert, bleibt in diesem Modell nebensächlich. Wie ich schon angedeutet habe, hielte ich es für einen Fehler, diese Strukturüberlegungen ganz zu den Akten der Wissenschaftsgeschichte zu legen. Kritik wurde ja vielfach geübt, besonders stark von ELISABETH SCHMHD. ${ }^{39}$ Ihre Polemik schießt über das Ziel hinaus, aber man kann der Kritik SCHMIDs und anderer in dem Punkt beipflichten, dass die unergänzte ,Anwendung' der Doppelwegsstruktur als vermeintlicher Interpretationsschlüssel zu unbefriedigenden Ergebnissen führt. Der Blick auf die Figuren kann helfen, diese zu differenzieren. Es geht dabei nicht darum, die Schemagebundenheit der Figuren in Frage zu stellen, sondern die Spannungen auszuloten, die Schemagebundenheit und weitere Ebenen des Figurenentwurfs erzeugen können.

Zunächst ist festzuhalten, dass KuHNs Modell ganz entscheidend vom männlichen Protagonisten als Handelndem her konstruiert ist. Das ist im Grundsatz nicht ganz unbegründet, heißt es doch gleich zu Beginn des Fragments, dass es Erec sei, durb den die rede erhaben ist (V. 4; „um dessentwillen diese Erzählung begonnen wurde`). Erec ist der Held in ganz schematischem Sinne, und Enite kommt als die gegen Widerstände zu erringende schönste Frau erst später ins Spiel. Auch im letzten Teil der Handlung und vor allem in der Joie de la Curt-Episode steht Erec klar im Zentrum. Dagegen bleibt Enite in KUHNs Modell unkonturiert - und das, obwohl sie mit Beginn der mittleren Partie eine entscheidende Rolle zu spielen beginnt, die weit über ihre vermeintliche aktantielle Determination

37 Hugo KuHN: Erec (1948). In: Ders.: Dichtung und Welt im Mittelalter. Kleine Schriften 1, Stuttgart 1959, S. 133-150.

38 Die für KUHN zentrale sinnbildende Technik von Erec und Enite beschreibt WNLTER HAUG so: „Die Stationen der vorgegebenen Struktur werden [...] mit Fpisoden besetzt, die einerseits in linearer Verknüptung cinen Handlungszusammenhang ergeben, die aber andeterseits ihre eigentliche Bedeutung quer zu diesem Zusatmmenhang aus ihrer strukturellen Position beziehen." WALTER HAUG: Die Symbolstruktur des höfischen Epos und ihre

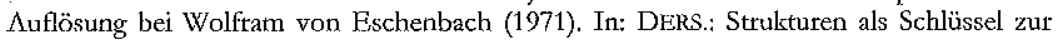
Welt. Kleine Schriften zur Erzählliteratur des Mittelalters, Tübingen 1989, S. 483-512, hier S. 484.

39 ELISABETH SCHMID: Weg mit dem Doppelweg. Wider eine Selbstverständlichkeit der getmanistischen $\Lambda$ rtusforschung. In: Erzählstrukturen in der Artusliteratur. Forschungsgeschichte und neue Ansätz.e. Hrsg. von FrIEDRICH WOLFZETTEL, Tübingen 1999, S. 69-85. 
(als zu erringende Frau) hinausgeht. ${ }^{40}$ Hartmanns Erec und Enite (ich wähle diesen Titel bewusst) hat ja spätestens mit dem Beginn des zweiten Teils, der aus der einfachen Märchenhandlung eine komplexere, problematischere macht, zwei Protagonisten.

Die Situation ist bekannt: Erec und Enite verligen sich, die Reputation des Königspaares fällt. Enite erhält davon Kenntnis, und klagt zu Mittag in der sonnendurchfluteten Kemenate, im Ehebett abgerückt von Erec, leise über die üble Nachrede, als sie ihren Mann schlafend glaubt. ${ }^{41} \mathrm{Erec}$, der die Klage hört, stellt seine Frau zur Rede und erfährt vom Reputationsverlust. Er agiert daraufhin sofort, rüstet sich heimlich und verlässt mit Enite den Hof, vorgebend am Abend wieder zurück zu sein, ohne dass die Motivation für sein Handeln und für die Täuschung gänzlich klar würde. Diese Unklarheit besteht für die Informationsvergabe an die Rezipienten ebenso wie für die Figuren auf der Ebene der Handlung. Eigens wird gesagt, dass kein Hofangehöriger Erecs Absichten begriffen hätte:

dô enwas aber niemen,

der sich des mohte verstân,

wie sîn gemüete was getân. (V. 3077-3079)

da gab es aber niemanden, der verstehen konnte, wie es in seinem Inneren aussah.

Erec befiehlt Enite vorauszureiten und verbietet ihr das Sprechen, ohne dass klar würde, worin dieses Verbot, das als Bestrafung semantisiert ist, begründet wäre: eine der großen unklaren und vielleicht nicht zur Klärung zu bringenden Forschungsfragen zu Erec und Enite. MARTIN JONES hat vor einigen Jahren in einem Aufsatz im Anschluss an NORRIS LACYs Interpretation von Chrétiens Erec et Enide darauf hingewiesen, dass dieșe entscheidende Krise im mittleren Teil des Romans auch deshalb so viele Interpretationsschwierigkeiten birgt, weil sich der point of view verschiebt: der point of view ist der Enides, und dieser Teil von Chrétiens Roman ist, anders als der etste und dritte Teil, aufgrund der narrativen Steuerung, Enides Geschichte. ${ }^{42}$ Hartmann folgt dieser Grundrichtung, ja scheint sie durch gewisse Techniken sogar noch zu akzentuieren. ${ }^{43}$

Eine entscheidende Spannung in der entsprechenden Partie des deutschen Textes wird über die Filterung der Innensichten erzeugt: Während

40 Das hat die Forschung längst aufgearbeitet: MEYJER, Struktur (Anm. 32); BRITTA BUSSMANN: Dô sprach diu edel künegîn ... . Sprache, Identität und Rang in Hartmanns Erc. In: ZfdA 134 (2005), S. 1-29; MANUEI.A NIESNER: scbiltkneht Enite. Zur: gendet-Transzendierung im Erec Hartmanns von Aue. In: ZfdPh 126 (2007), S. 1-20.

41 Zu dieser Szene Mưluer (Anm. 23), S. 292-296.

42 NORRrs J. LACY: The Craft of Chrétien de Troyes. An Essay on Narrative Art, Leiden 1980 (Davis Medieval Texts and Studies 3), S. 40-42; vgl. dazu anch JONES (Anm. 35), S. $300 \mathrm{f}$.

43 JONES ( $\Lambda \mathrm{nm} .35)$, S. 299-307. 
Enites muot, ihr seelisches Innere, vor allem über gesprochene und innere Monologe als Etfahrungstaum zugelassen ist, bleibt Erecs gemüete unzugänglich. Diese Unlesbarkeit bezieht sich nicht nur auf die anderen Figuren der Etzählwelt, sondern auch auf die Rezipienten des Textes: niemand am Hofe Erecs (Figurenebene), aber auch kein Rezipient ,blickt durch'.44 Auch später wird dies noch einmal deutlich markiert. Auf die Frage des ersten (namenlosen) Grafen, warum Erec seine Frau getrennt von ihm selbst essen lasse, antwortet er: herre, mîn gemüete stât alsô (V. 3745; „Herr, dies entspricht meinem inneren Befinden"). Die Formulierung unterstreicht die Innensichtrestriktion, an dieser Stelle sogar von der Figur selbst formuliert. Der. Text lässt Erec sagen, dass er so und nicht anders handle, weil dies seinem Inneren entspreche; über den (alles entscheidenden) inneren Zustand selbst aber erfährt der Fragende, und mit ihm Leser und Hörer, nichts.

In der mittleren Passage von Hartmanns Roman liegt also ein negativer Innensichtfilter vor (wir sehen nicht in Erec hinein), zusätzlich aber, das lässt sich in den der Krise folgenden Räuberepisoden zeigen, ist der Raumfilter der Enites. Es geht darum, wie Enite die bedrohliche Welt sieht und wie sie das durch das Redeverbot ausgelöste Dilemma und ihren Impuls, Erec zu warnen, bearbeitet. Die Fokalisierung hat also nicht nur den Effekt, dass Erecs Motivationen durch die Erzähltechnik gezielt verborgen werden, indem Enites point of view eingenommen wird. ${ }^{45}$ Vielmehr teilen Enite und das Publikum die beschränkte Sicht, das Etleiden und Nichtbegreifen von Erecs undurchsichtigem Handeln. Daher meine ich, dass der Text hier nicht dazu auffuft, Erecs Motivation aus den widersprüchlichen Informationen zu entschlüsseln. ${ }^{46}$ Vielmehr ist das Rätselhafte an Erecs Verhalten Programm. Er soll nicht gelesen werden.

In einem Punkt allerdings, was in den Diskussionen zu Erecs rätselaufgebendem Verhalten gerne übersehen wird, ist sein Verhalten glasklar: er zieht aus nâch aventiure wâne (V. 3111; ,,in der Hoffnung auf Aventiure“). Dieses schematische Handeln, der erneute Auszug und die Bewährung in Aventiuren zur Restitution der sozialen Ehre, wäre unproblematisch, wären da nicht Enite und ihre Behandlung. Erec erscheint in dieser Partie des Romans als handlungsfunktional bestimmter Aventiureritter. Er absolviert Stationen, treibt Handlung voran; seine Motivationen oder ein Innenleben werden nicht thematisiert. Erec ist in dieser Partie ganz auf die

44 LACY (Anm. 42), S. 40, beobachtet eine ähnliche Verweigetung der Einsicht in Erecs innere Motivationen in Chrétiens Erec et Enide; siehe dazu auch JONES (Anm. 35), S. $300 \mathrm{f}$.

45 So LACY (Anm. 42), S. 40, und JONES (Anm. 35), S. 300f.

46 So aber JONES ( $\Lambda \mathrm{nm}$. 35), S. 305-307, der meint, Etrecs Verhalten sei von den Rezipienten als ,pretence“ $z$ u deuten. 
Funktion der Handlungserzeugung reduziert. ${ }^{47}$ Das störend-verstörende Element ist, dass er die schönste Frau der Erzählwelt zum Pferdeknecht degradiert. Zum Verhalten Erecs gibt es explizite erklärende Textsignale, etwa die Äußerung Enites, dass sie den Ehrverlust als ihre Schuld begreift, die Aussage im nachhinein, dass Erec seine Frau prüfen wollte, und eben auch die Aussage, dass die Behandlung Enites âne sache (V. 6775) war, also keine Begründung hatte. ${ }^{48}$ Diese Textsignale sind aber uneindeutig, und verstärken in ihrer Widersprüchlichkeit eher die Ambivalenz und Unlesbarkeit von Erecs Verhalten, als dass sie es erklären. ${ }^{49}$ Die entwürdigende Behandlung Enites also bleibt unerklärt, und die befremdete Reaktion anderer Figuren der Textwelt auf diese Behandlung dient der textuellen Absicherung einer erwarteten oder erwartbaren Leser/Hörerreaktion, nämlich der Verstärkung der Meinung, dass Erecs Verhalten in der Tat befremdlich und auch nicht durch wie auch immer geartete mysogyne Sttukturen mittelaltetlicher Kultur gedeckt ist. INGRID KASTEN hält fest: „Erec wird anderen problematisch, nicht aber sich selbst“ ${ }^{\text {". }} .0$ Es zeigt sich auch daran, dass die Erzählersteuerung ebenfalls das Problematische an Erecs Verhalten hervorhebt: In der Tat ,legt der Erzähler nicht nur an dieser Stelle, sondern schon zuvor oft und deutlich genug gegen die Enite von Erec auferlegten Demütigungen sein Veto ein, so dass dieser alles andere ist als der ideale Repräsentant einer dem Mittelalter adäquaten Denkweise." $" 51$

Als Figur ist Enite selbst geradezu entgegengesetzt entworfen, und daraus lässt sich systematisch wie historisch einiges für eine narratologische Betrachtung der Figur ableiten. Von Enites Rede im Ehebett an wird regelmäßig eine privilegierte Sicht in ihr Inneres geboten. Es lässt sich daher eine gezielte Opposition zum Entwurf Erecs in dieser Partie vermuten. muot ist eine Art Leitwott im Sprechen über Enite. Des wart vil riunec ir muot (V. 3002, "darüber wurde sie in ihrem Inneren seht traurig"), heißt es zu Beginn der Krise. In Zwiespalt, ob sie Erec vor den Räubern warnen sollte, obwohl er ihr das Reden verboten hatte, redete si in ir muote (V. 3148;

47 JONES (Anm. 35) betont Erecs Empathiefähigkeit. Diese kann man aber, gerade im Vergleich mit Enite, nur sehr vereinzelt nachweisen.

48 Mit den Worten dem unbescheiden bazze wart ein ende gegeben (7099f.) lässt der Text sie sich nach dem zweiten Guivteiz-Kampf wieder vertragen. Das qualifizierende $\Lambda$ djektiv stützt die negative Beurteilung durch die Erzähletinstanz.

49 Vgl. auch das Referat der Forschungspositionen bei ScHOLZ (Anm. 27), S. 869-882, das zu einem ähnlichen Ergebnis kommt.

50 INGRD KASTEN: Bachtin und det böfssche Roman. In: bickelwort und mildit maere. Festschrift für Eberhard Nellmann zum 65. Geburtstag. Hrsg. von DORO'IHEE I.INDEMANN/BERNDT VOL.KMANN/KLAUS-PETER WEgerA, Göppingen 1995 (GAG 618), S. 51-70, hier S. 64.

51 ScHoLz (Anm. 27), S. 872 f. 
",redete sie in ihrem Inneren"). Gleich darauf wird mitgeteilt, welcher muot in Enites gedanc kam (V. 3167). Wohlgemerkt: Diese Aussagen fallen keine hundert Verse nach den oben zitierten Worten, dass die Natur von Erecs gemüete seinem Hof unklar sei. Enite, zum Schweigen gezwungen und bloß reaktiv, ist durch die undurchsichtigen Regeln ihres Mannes entmachtet und zu äußerlichem Schweigen verurteilt, aber spricht in ir muote. 52 Deutlich sichtbar wird dadurch der Gegensatz zwischen der Kreation und der Diskursivierung des muotes auf Enites Seite und der Restriktion der Innensicht in Erecs Fall.

Entscheidendes am Figurenentwurf der beiden Hauptfiguren ist daher nicht auf der bistoire-Ebene, sondern auf der discours-Ebene gestaltet. Auf der bistoire-Ebene haben Erec und Enite ja denselben Status. Erst in der konkreten Ausgestaltung, also auf der discours-Ebene, in der Enite eine neue Möglichkeit der Fokalisierung zugewiesen wird, wie sie sich im höfischen Roman des zwölften Jahrhunderts entwickelt, zeigt sich die entscheidende Differenz. Erecs Handlung bleibt unerklärt, er wird als schemagemäß Agierender präsentiert, als Dominanz suchender Ritter, der nâch âventiure wâne auszieht. Enite dagegen, äußerlich unfreier, darf im Inneren freier agieren, abwägen, entscheiden, sie wird gezeigt als eine Figur, die an den Möglichkeiten einer ,neuen Kultur der Innerlichkeit“53 im höfischen Roman partizipiert. Das ist nicht nur Abbildung von gegebenen und neu funktionalisierten historischen Möglichkeiten des Figurenentwurfs für den höfischen Roman, was an sich vielleicht ja schon ein interessanter Befund für eine historische Narratologie wäre. Es ist auch Illustration der Spannung zwischen schemagebundenem, objektivem' Handeln auf der einen Seite und durch Innenweltsicht neu motiviertem, subjelstiv begründetem Handeln auf der anderen. Das zweite wird in Ansätzen vorgeführt in der Darstellung des Dilemmas in Enites muote. In aller Deutlichkeit kann die Spannung aber nur durch den Gegensatz zwischen Erec und Enite gezeigt werden, und so wird Hartmanns Roman, oder zumindest dessen Mittelpassage, auch zu einer Reflexion auf erzählerische Möglichkeiten der Figurenkonzeption. Dieser Niederschlag konfligierender Anthropologien und letztlich auch Narratologien, zeichnet Hartmanns Erec und Enite sicherlich ebenso aus wie das Strukturschema. Strukturanalytische Studien wären daher zu ergänzen um die Spannungen, die Figurenentwürfe und ihre diskursive Präsentation bieten. Das hieße aber, dass die Spannungen, die

52 Zu Reden und Schweigen im Erec zusammenfassend BumKE (Anm. 34), S. 113-128 (mit der wichtigen Literatur S. 113). Nicht zustimmen kann ich BUMKEs Aussage: „Wenn man sagt, dass Reden und Schweigen das Hauptthema des Erec ist, dürfte man heute kaum noch Widerspiuch finden" (ebd.); Reden und Schweigen sind eher Symptome im Rahmen der Darstellung eines zentralen Problems als das Hauptthema des Textes. 
konfligierende textuelle Anthropologien in den Text hineinbringen, ein besonders fruchtbares Feld der Analyse zu sein scheinen.

\section{Konfligierende textuelle Anthropologien}

Die Analyse der Binnenpassage von Hartmanns Erec und Enite hat erbracht, dass zwei Figurenkonzepte vergleichsweise unvermittelt und spannungsreich nebeneinander stehen. Der narratologische Zugriff hilft, diese Spannungen klares: zu konturieren. Sie resultieren aus konfligierenden Realisationen von Möglichkeiten des Figurenentwurfs für den höfischen Roman, die gezielt gegeneinander gesetzt sind. Es versteht sich, dass dieses Beispiel nur eines unter vielen ist, die aus narratologischer Sicht zu vormodernen Figurenentwürfen analysiert werden könnten. Andere Felder liegen auf der Hand, etwa der Bereich der Schemainterferenzen und der Hybridität von Figuren. ${ }^{54}$ Auch für diese Fälle scheint es angemessen, nicht nach harmonisierenden Modellen der Figur zu suchen, sondern sich den Spannungen des Figurenentwurfs auszusetzen. Auch hier lässt sich vermuten, dass ein Modell bloßer Aktantenrealisation für die mittelalterliche literatur nicht ausreicht, man es sich aber gleichzeitig nicht leisten kann, die Schemagebundenheit von Handlungs- und Figurenentwurf auBer acht zu lassen.

Narrative Technik erzeugt im hier analysierten Fall eine Spannung zwischen den Entwürfen von Erec und Enite, gleichzeitig aber auch eine Spannung in den Figuren selbst. Die Anwesenheit der jeweils eine andere ,Anthropologie vertretenden Figur in derselben Erzählwelt setzt die jeweiligen Figurenentwürfe auch in sich, durch die Anwesenheit einer jeweils nicht tealisierten Alternative, in dialektische Schwingung. Sicher aber ist es nicht die Struktur, die die Handlungen der. Figuren vorgibt. Vielmehr ist deren Handeln von den narrativ erzeugten Grundbedingungen des Figurenentwurfs her erklärbar. Die narrative Kreation und Kundgabe von Enites seelischem Innenraum ist das entscheidende Element dieser ganzen Partie: Die dahinterstehende ,Anthropologie', nämlich dass Menschen

54 Dies wurde für den so genannten nachllassischen Roman im Blick auf die fehlende Kohätenz der Figurenentwürfe diskutiert. Siehe STEPHAN FUCHS-JOLIE: Hybride Helden. Gwigalois und Willebalm. Beitzäge zum Heldenbild und zur Poetik des Romans im frühen dreizehnten Jahrhundert, Heidelberg 1997 (Frankfurter Beiträge zur Germanistik 31); ARMIN SCHULZ: Poetik des Hybriden. Schema, Variation und intertextuelle Kombinatorik in der Minne- und Aventiureepik, Berlin 2000 (Philologische Studien und Quellen 161), S. 63-74; vgl. auch, zur sequentiellen Anordnung, VOIKJ MERTENS: Herrschaft, Buße, Liebe. Modelle adliger Identitätsstiftung in Mai und Beaflor. In: German Narrative Literature of the Twclth and Thirteenth Centuries. Essays Presented to Roy Wisbey on his Sixty-fifth Birthday. Hrsg. von VOL.KER HONIMANN u. a., Tübingen 1994, S. 391-410. 
einen verbal veräußerbaren Innentaum haben, der in Konflikt zur AuBenwelt stehen kann - die allgemein dem höfischen Roman zugesprochene Neuerung -, ist hier aber nicht unhinterfragte homogene Grundlage des Entwurfs, sondern findet ihren unmittelbaren Kontrast im akzentuiert und thematisiert schematischen Entwurf Erecs. Diese Pluralität zeugt von einer inhomogenen ,Anthropologie‘. Ein weiterer wichtiger Befund ist, dass diese narrative Möglichkeit der Innenraumdarstellung im höfischen Erzählen das schemabestimmte Handeln einer Figur, deren Motivation im Dunkeln bleibt, als Innensichtrestriktion und damit als Problem erscheinen läßt. In Gattungen und Traditionen, in denen Innensicht nicht prononciert vorgesehen ist, wäre solches Handeln als Normhandeln dagegen nicht per se problematisch.

Erec und Enite diskutiert implizit - durch narrative Technik - die Brüche zwischen den Figurenentwürfen. Die Verfügbarkeit der Entwürfe wird vor allem darin deutlich, dass an wenigen Stellen aufscheint, dass Erec eine gewisse Entscheidungsfreiheit über Innensichtrestriktionen (min gemiiete stât alsố, V. 3745) hat; er kann sein gemüete unlesbar bleiben lassen. So lässt sich hier wie an anderen epischen Texten des hohen Mittelalters nachweisen, dass Reflexe narrativer Entscheidungen auf die Figurenebene gespiegelt werden. Dies betont die Bedeutung, die in einer Modellierung einer Poetik des hochhöfischen Romans die Figur gegenüber Raum- und Zeitstrukturen hat. Figuren erscheinen so nicht als statische Handlungsträger (existents) deren Rolle vor allem darin besteht, das nächste Strukturereignis (event) zu ermöglichen. Vielmehr werden Struktur, Raum und Figur in einer Weise dynamisiert, dass die Durchlässigkeit zu einem Inneren (das in dieser Bewegung als solches allererst konstituiert wird) und die hermetische Abschließung desselben, wie sie uns bei Enite und Erec in der mittleren Partie von Hartmanns Roman begegnen, selbst zum entscheidenden Ereignis werden. Von hier aus ließe sich das Verhältnis von Figur und Erzählstruktur neu fundieren sowie das Verhältnis von existents und events überdenken. 